\title{
Red Mold Dioscorea Has Greater Hypolipidemic and Antiatherosclerotic Effect than Traditional Red Mold Rice and Unfermented Dioscorea in Hamsters
}

\author{
Chun-Lin Lee,${ }^{\dagger}$ Hsi-Kai Hung,${ }^{\dagger}$ Jyh-Jye Wang, ${ }^{\ddagger}$ And Tzu-Ming Pan $*, \dagger$ \\ Institute of Microbiology and Biochemistry, National Taiwan University, 1, Section 4, \\ Roosevelt Road, Taipei, Taiwan, R.O.C., and Department of Biotechnology, Tajen University, \\ Pingdon, Taiwan, R.O.C.
}

\begin{abstract}
Monascus-fermented red mold dioscorea (RMD) was proven to produce higher monacolin $\mathrm{K}$ levels than red mold rice (RMR) in our previous study. The goal of this study is to investigate whether the novel RMD had more hypolipidemic and antiatherosclerotic effect than traditional red mold rice. The daily dose of RMR for adults was recommended as $1 \mathrm{~g}$, which corresponded to $96 \mathrm{mg} / \mathrm{kg} / \mathrm{day}$ for hamsters. Therefore, high cholesterol diet-induced hyperlipidemic hamsters were daily administrated with a 0.5 -fold $(48 \mathrm{mg} / \mathrm{kg} /$ day), a 1 -fold $(96 \mathrm{mg} / \mathrm{kg} /$ day), or a 5 -fold dose $(480 \mathrm{mg} / \mathrm{kg} /$ day) of RMD for 8 weeks. Furthermore, a 1 -fold dose of RMR (96 mg/kg/day) and unfermented dioscorea (96 $\mathrm{mg} / \mathrm{kg} /$ day) were also respectively used to evaluate the effect of hypolipidemic and antiarteriosclerosis. The results indicated that only needing a 0.5 -fold dose of RMD was able to significantly lower total cholesterol (by $13.78 \%, p<0.001$ ), triglyceride (by $38.74 \%, p<0.01$ ), and low-density lipoprotein cholesterol levels (by $43.11 \%, p<0.05$ ) as well as maintain a high-density lipoprotein cholesterol level, as compared to the hyperlipidemic group. RMD including a higher monacolin $\mathrm{K}$ level and a dioscorea substrate was able to exhibit a more significant difference in the hypolipidemic effect than RMR or unfermented dioscorea. Both RMR and dioscorea exhibited potent in vitro antioxidative ability and in vivo protection against hypolipidemia-induced oxidative stress. Therefore, the antioxidative ability of RMD provided by Monascus metabolites (dimerumic acid, tannin, phenol, etc.) as well as dioscorea was able to perform more antiatherosclerotic effects on increasing total antioxidant status, catalase, and superoxide dismutase activity and repressing lipid peroxidation and atherosclerotic plaque than RMR and dioscorea.
\end{abstract}

KEYWORDS: Monascus; red mod rice; red mold dioscorea; dioscorea; monacolin; hypolipidemia; antiatherosclerosis

\section{INTRODUCTION}

Cholesterol is always regarded as the risk factor for the development of cardiovascular diseases. There is compelling evidence that oxidized lipoproteins are atherogenic and play a key role in the pathogenesis of coronary heart disease (1). Oxidized lipoproteins have been identified in atherosclerotic lesions in both animals and humans (2). Therefore, developing a potent drug or functional food with lowering cholesterol and repressing oxidized lipoprotein formation is an important topic in current studies.

The Monascus species has been used as a traditional food fungus in Eastern Asia for several centuries. Since the worthful secondary metabolite-monacolin $\mathrm{K}$ was proven as the inhibitor of HMG-CoA reductase in the cholesterol biosynthesis pathway,

* Corresponding author phone: +886-2-3366-4519 ext 10; fax: +8862-2362-7044; e-mail: tmpan@ntu.edu.tw.

National Taiwan University.

Tajen University.
Monascus fermented rice known as red mold rice (RMR) was gradually developed as a popular functional food for hypolipidemia $(3,4)$. Many RMR-related commercial products such as Cholestin have been applied to prevent hyperlipidemia for many years, and some research also reported its antiatherosclerosis effect and mechanism $(5,6)$. Monacolin K of RMR is proven to lower the level of serum low-density lipoprotein cholesterol (LDL-C) and inhibit the formation of ox-LDL, which would further prevent the occurrence of atherosclerotic lesions (5). Our previous study found that higher monacolin K production is able to be obtained in a novel and worthful Monascus-fermented product-red mold dioscorea (RMD) (3). According to the proven hypolipidemic mechanism of RMR, RMD including higher monacolin $\mathrm{K}$ should provide a more potent hypocholesterolemic effect. In addition, monascin, a Monascus yellow pigment with anti-inflammatory potential, is significantly formed and substituted for the red pigment (monascorubramine and rubropunctamine) as the major pigment of RMD (7). The anti- 
inflammatory ability is also proven to prevent the occurrence of atherosclerotic lesions (8).

RMD comprises a dioscorea root substance besides Monascus metabolite. The dioscorea root is regarded as a functional food or a worthful herb because of the inclusion of many functional ingredients for the prevention of various diseases (9). Dioscorin, polysaccharides, flavones, vitamin $\mathrm{C}$, polyphenol, and sporamin of dioscorea are proven to exhibit great antioxidative ability $(10-12)$, which should be of great benefit to blockade the modification of oxidized-low-density lipoprotein (ox-LDL) (13). In addition, dioscin and diosgenin of dioscorea are proven to have anti-inflammatory and hypolipidemic abilities $(11,12)$. Therefore, the function of dioscorea should strengthen the hypolipidemic and antiatherosclerotic effect of RMD.

The goal of this study is to evaluate whether RMD performs a more potent hypolipidemic and antiatherosclerotic effect than RMR. In this study, monacolin $\mathrm{K}$ and yellow pigment levels and the antioxidative ability of RMD and RMR were determined and compared in the in vitro evaluation. The in vivo hypolipidemic and antiatherosclerotic effect of RMD, RMR, and dioscorea was further compared and investigated in the hyperlipidemic hamster. The serum and liver lipid levels were used as the markers of hypolipidemic effect. The effect of antioxidase activity and the repressing lipid peroxidation and atherosclerotic lesion were used to evaluate the antiatherosclerotic effect.

\section{MATERIALS AND METHODS}

Chemicals. Monacolin K (mevanolin), thiobarbituric acid (TBA), and malondialdehyde (MDA) were purchased from Sigma Chemical Co. (St. Louis, MO). LC grade acetonitrile, chloroform, methanol, and dimethyl sulfoxide (DMSO) were purchased from Merck Co. (Darmstadat, Germany). Tryptone, yeast extract, peptone, malt extract, PDA broth, and Bacto-agar were purchased from Difco Co. (Detroit, MI).

Preparation of Red Mold Dioscorea and Red Mold Rice. Monascus purpureus NTU 568 was a mutant with high monacolin K productivity; its RMR product has been proven to perform a potent hypolipidemic effect in our previous study (3). The culture strain was maintained on potato dextrose agar (PDA) slanted at $10{ }^{\circ} \mathrm{C}$ and transferred monthly. The dioscorea root (Dioscorea batatas Dence) purchased from a local supermarket in Taiwan was used to produce red mold dioscorea using the method of solid-state culture (7). The preparation of RMR was carried out under the substrate of long-grain rice (Ipomoea batatas) purchased from a local supermarket in Taiwan using the method of solid-state culture (14). Briefly, a $500 \mathrm{~g}$ substrate was soaked in distilled water for $8 \mathrm{~h}$. After that, excess water was removed with a sieve. The substrate was autoclaved for $20 \mathrm{~min}$ at 121 ${ }^{\circ} \mathrm{C}$ in a "koji-dish" (the koji-dish was made of wood with the dimensions of $30 \mathrm{~cm} \times 20 \mathrm{~cm} \times 5 \mathrm{~cm}$ ). After having been cooled, the substrate was inoculated with a $5 \%(\mathrm{v} / \mathrm{w})$ spore suspension. The inoculated substrate was cultivated at $30{ }^{\circ} \mathrm{C}$ for 10 days. During the culturing stage, $100 \mathrm{~mL}$ of water was daily added to the substrate from the second day to the fifth day. At the end of cultivation, the crushed and dried product with the mold was used for the experiments (14).

Animals and Diets. Sixty-four male Golden Syrian hamsters weighing 100-120 g were housed in individual plastics cages and subjected to a 12-h light/dark cycle with a maintained relative humidity of $60 \%$ and a temperature at $25^{\circ} \mathrm{C}$. The animals were given free access to regular rodent chow and water for 4 weeks in order to adapt to the new environment. Hamsters were weighed and randomly assigned to 8 groups of 8 animals each before the commencement of the animal experiment.

Dose and Grouping. The dose of RMD powder was calculated in accordance with Boyd's Formula of Body Surface Area as recommended by the FDA (Food and Drug Administration) (15). The daily dietary dose of commercial Monascus product is usually recommended and used at $1.0-2.0 \mathrm{~g}$ for an adult $(6,16)$. Using $1 \mathrm{~g}$ of RMR as the reference dose of an adult to calculate the hamster dose has been proven to exhibit a hypolipilidemic effect in our previous study (3). Probucol used as a positive hypolipilidemic drug also has a recommended dose of $1 \mathrm{~g}$ /day for an adult (17). Therefore, RMD, RMR, dioscorea, and probucol are used as the reference dose at $1 \mathrm{~g}$ for an adult with a weight of $65 \mathrm{~kg}$ and a height of $170 \mathrm{~cm}$. These dosages were used as a frame of reference for the conversion of the dose into a hamster model. Therefore, feeding a hamster with a 0.5 -fold dose of RMD per day corresponds to supplementing the daily diet with $0.5 \mathrm{~g}$ of RMD for an adult. After the prebreeding stage for 4 weeks, all test samples were respectively suspended in $1 \mathrm{~mL}$ of water and orally administrated to the hamsters using a stomach tube for 8 weeks. The food intake was recorded daily, and animals were weighed weekly.

Experimental diets were provided in accordance with AIN-76 diet formulation with modification (American Institute of Nutrition, 1977). The control group was fed a normal diet via AIN-76 formulation, and the $\mathrm{H}$ group was given a high cholesterol diet that contained $0.2 \%$ cholesterol $(3,18)$. H-RMD0.5X, H-RMD1X, and H-RMD5X groups were fed the high cholesterol diet and orally given a 0.5 -fold dosage of RMD (48 mg/kg BW per day including $0.84 \mathrm{mg}$ of monacolin K), a 1-fold dose of RMD (96 mg/kg BW per day including $1.68 \mathrm{mg}$ of monacolin K), and a 5 -fold dose of RMD (480 mg/kg BW per day including $8.4 \mathrm{mg}$ of monacolin $\mathrm{K}$ ), respectively. In addition, the H-PBC group, a positive control group, was fed the high cholesterol diet and given orally a 1 -fold dose of probucol $(100 \mathrm{mg} / \mathrm{kg} \mathrm{BW}$ per day). To investigate and clarify whether the effect of RMD on the hypolipidemic and antiatherosclerotic effect is more than that of RMR and unfermented dioscorea, the same doses of RMD, RMR, and unfermented dioscorea were fed to the hyperlipidemic hamsters, respectively. The H-RMR1X group was fed with a 1 -fold dose of RMR $(96 \mathrm{mg} / \mathrm{kg}$ BW per day including $0.83 \mathrm{mg}$ of monacolin $\mathrm{K}$ ) and the $\mathrm{H}$-DC1X group was fed with a 1-fold dose of dioscorea ( $96 \mathrm{mg} / \mathrm{kg} \mathrm{BW}$ per day). Furthermore, the H-RMD0.5X group and the H-RMR1X group were fed with an almost equal level of monacolin $\mathrm{K}$.

Twenty-four $\mathrm{h}$ before sacrifice, all food was removed. Animals were anesthetized and sacrificed by carbon dioxide inhalation, and whole blood, plasma, and serum samples were collected, prepared, and then stored at $-80{ }^{\circ} \mathrm{C}$. Liver tissue was lavaged and rinsed frequently with a $0.8 \%$ sodium chloride solution for eliminating any blood. The biggest leaf of liver tissue was ground in ice-cold phosphate buffer saline (PBS) and then centrifuged $(8000 \times \mathrm{g}, 15 \mathrm{~min})$. The supernatant was collected and stored at $-80{ }^{\circ} \mathrm{C}$ for the assay of superoxide dismutase (SOD) activity and thiobarbituric acid reactive substances (TBARS). The other liver tissue was immersed in the liquid nitrogen and then stored at -80 ${ }^{\circ} \mathrm{C}$. The experiment was reviewed and approved by the Animal Care and Research Ethics Committee of the National Taiwan University.

Serum and Liver Lipid Analysis. Serum TC, TG, and high-density lipoprotein cholesterol (HDL-C) levels were measured in triplicate using commercial enzymatic kits. These kits were as follows: the TC assay kit (CH 200, Randox Laboratories Ltd., Antrim, U.K.), the TG assay kit (TR-210, Randox Laboratories Ltd), and the HDL-C assay kit (CH-203, Randox Laboratories Ltd). Serum low-density lipoprotein cholesterol (LDL-C) levels were gained via the following calculation (19): LDL-C $(\mathrm{mg} / \mathrm{dL})=\mathrm{TC}-\mathrm{TG} / 5-\mathrm{HDL}-\mathrm{C}$.

Liver tissue $(0.5 \mathrm{~g})$ was ground in $10 \mathrm{~mL}$ of ice-cold Folch solution (chloroform: methanol $=2: 1 ; \mathrm{v} / \mathrm{v}$ ) and incubated for $30 \mathrm{~min}$ at room temperature. The aqueous layer was aspirated and discarded, and the fixed volume of the organic layer was then evaporated to dryness. The dried lipid layer was dissolved with an equal volume of DMSO and then used to determine the TC and TG levels using commercial enzymatic kits.

Determination of TBARS Content, Total Antioxidant Status (TAS), SOD Activity, and Catalase Activity. The TBARS assay is also regarded as the accepted determination for in vivo lipid peroxidation $(20,21)$. According to the procedure of the previous study (22), the TBARS levels of serum and liver were determined by the method of thiobarbituric acid (TBA) colorimetric analysis, and the optical density (OD) value was measured at $532 \mathrm{~nm}$. The homogenate supernatants of liver and erythrocyte were examined by the SOD activity using a commercial kit (Ransod, Randox Laboratories Ltd.). Determination of TAS of plasma was carried out using a commercial kit (TAS-NX2332, Randox Laboratories Ltd.). The assay principle is that metmyoglobin reacts with $\mathrm{H}_{2} \mathrm{O}_{2}$ to form the radical species, ferrylmyo- 
Table 1. Monacolin K, Yellow Pigments, and Antioxidant Activity in Red Mold Dioscorea (RMD), Red Mold Rice (RMR), and Unfermented Dioscorea $a^{a, b}$

\begin{tabular}{|c|c|c|c|c|c|}
\hline \multirow[b]{2}{*}{ sample } & \multicolumn{2}{|c|}{ Monascus secondary metabolite } & \multicolumn{3}{|c|}{ antioxidative ability } \\
\hline & $\begin{array}{l}\text { monacolin } \mathrm{K} \\
(\mathrm{mg} / \mathrm{g})\end{array}$ & $\begin{array}{c}\text { yellow pigment } \\
\left(A_{400} / \mathrm{g}\right)\end{array}$ & $\begin{array}{c}\text { DPPH radical } \\
\text { scavenging ability (\%) }\end{array}$ & $\begin{array}{l}\text { conjugate dienes } \\
\text { inhibition (\%) }\end{array}$ & $\begin{array}{c}\text { reducing } \\
\text { power }\left(A_{700}\right)\end{array}$ \\
\hline $\begin{array}{l}\text { RMD } \\
\text { RMR } \\
\text { dioscorea } \\
\text { rice } \\
\text { vitamin C }\end{array}$ & $\begin{aligned} 17.50 & \pm 0.79 \\
8.62 & \pm 0.32^{* * * *}\end{aligned}$ & $\begin{array}{l}115.23 \pm 3.82 \\
106.24 \pm 4.65^{*}\end{array}$ & $\begin{array}{l}74.56 \pm 4.27 \\
52.50 \pm 3.23^{* *} \\
81.20 \pm 1.74^{*} \\
84.17 \pm 2.16^{* *}\end{array}$ & $\begin{array}{l}86.01 \pm 1.74 \\
84.82 \pm 5.98 \\
84.15 \pm 0.12 \\
92.67 \pm 2.52^{* *}\end{array}$ & $\begin{array}{l}2.15 \pm 0.05 \\
2.47 \pm 0.11^{*} \\
1.93 \pm 0.09^{*} \\
0.80 \pm 0.01^{* *} \\
2.45 \pm 0.06^{*}\end{array}$ \\
\hline
\end{tabular}

\footnotetext{
${ }^{a}$ One gram of sample was extracted with $10 \mathrm{~mL}$ of Tris- $\mathrm{HCl}$ buffer $(0.05 \mathrm{M}, \mathrm{pH} 7.4)$ at $37^{\circ} \mathrm{C}$ for $60 \mathrm{~min}$. After centrifugation $(5500 \times \mathrm{g}, 10 \mathrm{~min})$, the supernatant was collected for the determination of antioxidative ability. ${ }^{b}$ Data were presented with mean $\pm S D(n=3) .{ }^{*} p<0.05,{ }^{* *} p<0.01$, and ${ }^{* * *} p<0.001$ versus the RMD group.
}

globin. A chromogen (2,2'-azinodi[ethylbenzthiazoline sulfonate]; ABTS) is incubated with the ferrylmyoglobin to produce the radical cation species ABTS. This has a relatively stable blue-green color, which is measured at $600 \mathrm{~nm}$. Antioxidants cause suppression of this color production to a degree that is proportional to their concentration (23). The catalase activity of the homogenate supernatant of liver and serum was determined as previously described (24). Catalase activity was monitored by the disappearance of $10 \mathrm{mM} \mathrm{H}_{2} \mathrm{O}_{2}$ by measuring the changes in absorbance at $240 \mathrm{~nm}$ for $3 \mathrm{~min}$.

Stain of Aortic Plaque in Artery. The thoracic aorta was cut open longitudinally along the anterior side, and the lipid-rich lesions on the surface of the aorta were stained with $2 \%$ Sudan IV and then successively washed with a gradient concentration of methanol $(100 \%$, $90 \%, 80 \%, 70 \%, 60 \%)$ and PBS. The whole surface area of thoracic aorta was stained by Sudan IV and photographed using a digital camera. The aortic surface area and its stained plaque area (red) were selected and quantitated by the Posterize program of Photoshop 7.0 software (Adobe Systems Incorporated, San Jose, CA). The selected pixel of the plaque area and whole aorta was used to calculate the percent area of the aortic plaque, as follows:

aortic plaque percent $(\%)=$

pixel of stained plaque area/pixel of whole aorta $\times 100 \%$

Determination of the Monacolin K Level of RMR and RMD. One gram of dried RMR or RMD samples was extracted with $10 \mathrm{~mL}$ of ethanol at $65^{\circ} \mathrm{C}$ for $30 \mathrm{~min}(25)$. The extracts $(10 \% \mathrm{w} / \mathrm{v})$ were further filtered with a $0.45 \mu \mathrm{m}$ filter and analyzed by HPLC. HPLC was performed according to the method described previously (25) and carried out on an HPLC system PU2089 plus (Jasco Co., Tokyo, Japan). A Discovery $\mathrm{C}_{18}$ column, $25 \mathrm{~cm} \times 4.6 \mathrm{~mm}$ i.d., $5 \mu \mathrm{m}$ (Bellefonte, PA), was used as the analytical column. The mobile phase consisting of $45 \%$ water, $55 \%$ acetonitrile, and $0.5 \%$ trifluoroacetate was eluted at a flow rate of $1.0 \mathrm{~mL} / \mathrm{min}$. Total monacolin $\mathrm{K}$ was detected using a UV detector UV2075 plus (Jasco Co.) set at $238 \mathrm{~nm}$ (25). The intraand interday CV values were less than $6.23 \%$ and $7.40 \%$, respectively. In addition, the $R^{2}$ value was 0.9914 .

Pigment Estimation. One gram of RMR or RMD was extracted with $95 \%$ ethanol at $37{ }^{\circ} \mathrm{C}$ for $90 \mathrm{~min}$. The supernatant including the yellow pigment was estimated using a spectrophotometer set at 400 $\mathrm{nm}$. The yellow pigment level was expressed as optical density units per gram of dried medium multiplied by the dilution factor (26).

In Vitro Examination of the Antioxidative Ability of RMD, RMR, and Unfermented Dioscorea. One gram of sample was extracted with $10 \mathrm{~mL}$ of Tris-HCl buffer $(0.05 \mathrm{M}, \mathrm{pH} 7.4)$ at $37^{\circ} \mathrm{C}$ for $60 \mathrm{~min}$. After centrifugation $(5500 \times \mathrm{g}, 10 \mathrm{~min})$, the supernatant was collected for the determination of antioxidative ability. The 2,2-diphenyl-1-picrylhydrazyl (DPPH) radical scavenging method proposed by Aniya et al. (27) was modified as follows: The reaction mixture consisted of 1 $\mathrm{mL}$ of $0.1 \mathrm{mM}$ DPPH in ethanol, $0.95 \mathrm{~mL}$ of $0.05 \mathrm{M}$ Tris-HCl buffer (pH 7.4), $1 \mathrm{~mL}$ of ethanol, and $50 \mu \mathrm{L}$ of sample or deionized water (control). The absorbance of the mixture was measured at $517 \mathrm{~nm}$ exactly $30 \mathrm{~min}$ after adding the extract. The reducing power was determined using the method proposed by Oyaizu (28). An amount of a 2.5 - $\mathrm{mL}$ sample $(0.25 \mathrm{~g} / \mathrm{mL})$ was mixed with phosphate buffer $(2.5$ $\mathrm{mL}, 0.2 \mathrm{M}, \mathrm{pH} 6.6)$ and potassium ferricyanide $(2.5 \mathrm{~mL}, 1 \%)$. The mixture was incubated at $50{ }^{\circ} \mathrm{C}$ for $20 \mathrm{~min}$ and then mixed with 2.5 $\mathrm{mL}$ of $10 \%$ trichloroacetic acid. After centrifugation $(5500 \times \mathrm{g}, 10$ $\mathrm{min})$, the upper layer of the solution $(5 \mathrm{~mL})$ was mixed with distilled water $(5 \mathrm{~mL})$ and $\mathrm{FeCl}_{3}(1 \mathrm{~mL}, 0.1 \%)$, and the absorbance of the resulting mixture was measured at $700 \mathrm{~nm}$. Increased absorbance of the reaction mixture implied increased reducing power. Conjugate dienes inhibition was determined using the method proposed by Lingnert et al. (29). The substrate consisting of linoleic acid was emulsified with an equal amount of Tween 20 in the phosphate buffer (0.02 M, pH 6.6). The $0.1-\mathrm{mL}$ sample was mixed with $2 \mathrm{~mL}$ of emulsion and then incubated at $50{ }^{\circ} \mathrm{C}$ for $48 \mathrm{~h}$, respectively. The formation of conjugated dienes was monitored and measured at $234 \mathrm{~nm}$. Decreased absorbance of the reaction mixture implied restraints on the formation of a conjugated diene from linoleic acid.

Statistics. Data are expressed as the mean \pm SD. The statistical significance in the behavioral and biochemical effects was determined by one-way analysis of variance (ANOVA), followed by ANOVA with the Scheffe test: ${ }^{*} p<0.05,{ }^{* *} p<0.01$, and ${ }^{* * *} p<0.001$ versus the $\mathrm{H}$ group; ${ }^{\#}<<0.05,{ }^{\# \#} p<0.01$, and ${ }^{\# \# \#} p<0.001$ versus the H-RMR1X group; and ${ }^{\dagger} p<0.05,{ }^{\dagger \dagger} p<0.01$, and ${ }^{\dagger \dagger} p<0.001$ versus the H-DC1X group.

\section{RESULTS}

The Profile of Monacolin K and Yellow Pigment Levels and the Antioxidative Ability in the RMD, RMR, and Unfermented Dioscorea. Table 1 indicated that RMD included more levels of monacolin $\mathrm{K}$ and yellow pigment than RMR as well as performed antioxidative ability as potent as dioscorea and vitamin C. RMR has a weaker effect on the DPPH radical scavenging ability than RMD in addition to fewer levels of monacolin $\mathrm{K}$ and yellow pigment. Although dioscorea is a lack of monacolin $\mathrm{K}$ and yellow pigment, the various active ingredients of dioscorea root have been proven to perform a remarkable hypolipidemic and antiatherosclerotic effect (12). According to the above results, RMD included the active compounds and the antioxidative ability of RMR and dioscorea, but the hypolipidemic and antiatherosclerotic effect of RMD has never been investigated. Therefore, the in vivo effect of RMD will be further investigated in this study.

The Change of Body Weight and Daily Intake by Hamsters. The results indicate that the body weight and daily intake of the hamsters increased normally and had no difference among the various groups during the period of the practical experiment (data not shown). In addition, the externals and health of all experimental animals had a normal expression.

Hypolipidemic Effect. Hypocholesterolemic Effect in Serum. As shown in Table 2, a high cholesterol diet would increase the serum TC level of the $\mathrm{H}$ group by $76.8 \%(p<0.001)$, as compared with the $\mathrm{C}$ group. However, the TC levels of the H-RMD0.5X group, the H-RMD1X group, and the H-RMD5X group were less by $17.8 \%, 21.3 \%$, and $20.8 \%$ than that of the 
Table 2. Hypolipidemic Effect of Red Mold Dioscorea (RMD) in the Serum of Hyperlipidemic Hamsters ${ }^{a}$

\begin{tabular}{|c|c|c|c|c|c|}
\hline groups & $\mathrm{TC}(\mathrm{mg} / \mathrm{dL})$ & $\mathrm{TG}(\mathrm{mg} / \mathrm{dL})$ & $\mathrm{HDL}-\mathrm{C}(\mathrm{mg} / \mathrm{dL})$ & LDL-C (mg/dL) & LDL-C/HDL-C ratio \\
\hline C & $130.14 \pm 7.78^{* * *} \# \#+1 \dagger$ & $61.33 \pm 15.72^{* \star \star}$ & $82.1 \pm 14.5^{*}$ & $43.1 \pm 15.4^{* \star *}$ & $0.53 \pm 0.16$ \\
\hline $\mathrm{H}$ & $230.05 \pm 26.80$ \#\#+† & $124.14 \pm 23.06^{\# \dagger}$ & $128.0 \pm 16.8^{\dagger}$ & $103.5 \pm 39.4^{\#}$ & $0.82 \pm 0.32^{\#}$ \\
\hline $\mathrm{H}-\mathrm{PBC}$ & $216.09 \pm 8.61^{\dagger}$ & $96.27 \pm 24.92$ & $119.1 \pm 31.7$ & $56.0 \pm 16.3^{*}$ & $0.50 \pm 0.18$ \\
\hline H-DC1X & $190.23 \pm 6.43^{* * *}$ & $102.03 \pm 13.17$ & $96.8 \pm 20.1^{*}$ & $71.1 \pm 25.8$ & $0.73 \pm 0.26$ \\
\hline H-RMR1X & $198.35 \pm 5.79^{* *}$ & $77.01 \pm 15.90^{*}$ & $117.3 \pm 24.8$ & $50.9 \pm 12.6^{* *}$ & $0.45 \pm 0.16^{*}$ \\
\hline H-RMD0.5X & $189.11 \pm 5.75^{\star * *}$ & $76.05 \pm 14.46^{* *}$ & $121.2 \pm 10.2$ & $58.6 \pm 14.4^{*}$ & $0.48 \pm 0.12$ \\
\hline H-RMD1X & $181.10 \pm 11.0^{* * * \#}$ & $71.24 \pm 28.46^{* *}$ & $121.0 \pm 25.8$ & $46.6 \pm 20.4^{* * *}$ & $0.39 \pm 0.19^{* *}$ \\
\hline H-RMD5X & $182.18 \pm 10.60^{* * \#}$ & $75.02 \pm 25.05^{* *}$ & $118.4 \pm 12.9$ & $51.1 \pm 7.4^{* *}$ & $0.44 \pm 0.09^{*}$ \\
\hline
\end{tabular}

\begin{abstract}
${ }^{a}$ Two groups of the hamsters were fed a normal diet (the $\mathrm{C}$ group) or a high cholesterol diet (the $\mathrm{H}$ group) without the administration of test materials, respectively. The other hyperlipidemic hamsters were administrated with probucol (100 mg/kg/day) (the H-PBC group), a l-fold dose of unfermented dioscorea (96 mg/kg/day) (the H-DC group), a l-fold dose of RMR ( $96 \mathrm{mg} / \mathrm{kg} /$ day including $0.83 \mathrm{mg}$ of monacolin K) (the H-RMR1X group), a 0.5-fold dose of RMD (48 mg/kg/day including $0.84 \mathrm{mg}$ of monacolin $\mathrm{K}$ ) (the H-RMD0.5X group), a 1-fold dose of RMD (96 mg/kg/day including $1.68 \mathrm{mg}$ of monacolin K) (the H-RMD1X group), and a 5 -fold dose of RMD (480 mg/kg/day including $8.4 \mathrm{mg}$ of monacolin $\mathrm{K}$ ) (the H-RMD5X group). ${ }^{*} p<0.05,{ }^{* *} p<0.01$, and ${ }^{* * * *} p<0.001$ versus the $\mathrm{H}$ group; $\# p<0.05$, \#\# $p<0.01$, and $\# \# p<0.001$ versus the $\mathrm{H}$-RMR1X group; ${ }^{\dagger} p<0.05,{ }^{\dagger \dagger} p<0.01$, and ${ }^{+t \dagger} p<0.001$ versus the $\mathrm{H}-\mathrm{DC} 1 \mathrm{X}$ group $(n=8)$.
\end{abstract}

$\mathrm{H}$ group $(p<0.001)$. Although a 1-fold dose of RMR performed the hypocholesterolemic effect by $13.8 \%$, its statistical significant effect ( $p<0.01$ vs the $\mathrm{H}$ group) was weaker than a halffold dose or a 1-fold dose of RMD ( $p<0.001$ vs the H group). It implied that RMD would lower more TC levels than RMR even though their dose of the monacolin $\mathrm{K}$ level was equal. In addition, unfermented dioscorea also performed a significant hypocholesterolemic effect of $p<0.001$ (vs the $\mathrm{H}$ group), which would be more than the statistical result of the RMR treatment ( $p<0.01$ vs the $\mathrm{H}$ group) and equal to the result of the RMD treatment ( $p<0.001$ vs the $\mathrm{H}$ group).

Hypotriglyceridemic Effect in Serum. The $\mathrm{H}$ group fed with a high cholesterol diet had a higher serum TG level by $102.4 \%$ than the $\mathrm{C}$ group $(p<0.001)$. The serum TG level would be significantly decreased by $42.6 \%$ in the H-RMD1X group $(p<0.01)$ and by $38.0 \%$ in the H-RMR1X group $(p<0.05)$. Therefore, the statistical significant difference between the H-group and the H-RMD1X group $(p<0.01)$ was more than that between the H-group and the H-RMR1X group $(p<0.05)$. The results indicated that administrating dioscorea to a hyperlipidemic rat was a default to significantly decrease the serum TG level $(p>0.05)$. Therefore, the hypotriglyceridemic effect of dioscorea was less than that of RMD, as based on the comparison with the TG level of the $\mathrm{H}$ group.

The Effect on HDL-C, LDL-C, and LDL-C/HDL-C. As shown in Table 2, the HDL-C level of the $\mathrm{H}$ group would be increased by $55.9 \%$ with increasing the TC level $(p<0.05)$. However, all RMD administrated groups had no significant decrease in the HDL-C level as compared with the H group $(p>0.05)$. The HDL-C level of the H-RMR1X group was slightly less by $8.4 \%$ than the $\mathrm{H}$ group but without a significantdifference $(p>0.05)$. A significant decrease by $24.4 \%$ in the HDL-C level was found in the H-DC group as compared with the $\mathrm{H}$ group $(p<0.05)$. Regarding the effect on the LDL-C level, feeding a high cholesterol diet to the $\mathrm{H}$ group resulted in a significantly higher LDL-C level than the $C$ group $(p<0.001)$. The LDL-C level would be significantly decreased by $50.8 \%$ in the H-RMR1X group $(p<0.01)$ and by $55.0 \%$ in the H-RMD1X group $(p<0.001)$ as compared to the $\mathrm{H}$ group. According to the statistical comparison between the test group and the $\mathrm{H}$ group, a 1-fold dose of RMD $(p<0.001$ vs the $\mathrm{H}$ group) has a stronger significant difference on the effect of lowering the LDL-C level than the same dose of RMR $(p<0.01$ vs the $\mathrm{H}$ group). Furthermore, treatment with unfermented dioscorea was the default to decrease the LDL-C level with a significant difference $(p>0.05)$. The ratio of LDL-C to HDL-C is another criterion for the development of cardiovascular diseases. If the ratio is low, then the TC level would include a
Table 3. Hypocholesterolemic and Hypotriglyceridemic Effect of Red Mold Dioscorea (RMD) in the Serum of Hyperlipidemic Hamsters ${ }^{a}$

\begin{tabular}{lcl}
\hline \multicolumn{1}{c}{ groups } & TC (mg/g liver) & TG (mg/g liver) \\
\hline C & $50.1 \pm 17.6^{* * \#+}$ & $11.8 \pm 0.7^{* *+\# \dagger}$ \\
H & $104.3 \pm 16.1$ & $20.3 \pm 1.8^{\#}$ \\
H-PBC & $111.5 \pm 32.1$ & $22.5 \pm 1.0^{\# \dagger \dagger}$ \\
H-DC1X & $104.0 \pm 33.3$ & $18.4 \pm 2.4^{\#}$ \\
H-RMR1X & $90.3 \pm 23.0$ & $15.1 \pm 1.5^{* \dagger}$ \\
H-RMD0.5X & $88.2 \pm 11.3$ & $14.2 \pm 1.5^{* \dagger}$ \\
H-RMD1X & $81.9 \pm 17.5^{*}$ & $13.6 \pm 3.1^{* * \dagger}$ \\
H-RMD5X & $82.7 \pm 14.3^{*}$ & $12.0 \pm 2.6^{* * \dagger}$
\end{tabular}

\begin{abstract}
${ }^{a}$ Two groups of the hamsters were fed a normal diet (the $\mathrm{C}$ group) or a high cholesterol diet (the $\mathrm{H}$ group) without the administration of test materials, respectively. The other hyperlipidemic hamsters were administrated with probucol (100 mg/kg/day) (the H-PBC group), a l-fold dose of unfermented dioscorea (96 $\mathrm{mg} / \mathrm{kg} /$ day) (the H-DC group), a l-fold dose of RMR (96 mg/kg/day including 0.83 $\mathrm{mg}$ of monacolin K) (the H-RMR1X group), a 0.5 -fold dose of RMD (48 mg/kg/day including $0.84 \mathrm{mg}$ of monacolin $\mathrm{K}$ ) (the H-RMD0.5X group), a 1-fold dose of RMD (96 mg/kg/day including $1.68 \mathrm{mg}$ of monacolin K) (the H-RMD1X group), and a 5 -fold dose of RMD (480 mg/kg/day including $8.4 \mathrm{mg}$ of monacolin K) (the H-RMD5X group). $" p<0.05, " * p<0.01$, and ${ }^{* * * *} p<0.001$ versus the $H$ group; $" \#<0.05$ and ${ }^{\#} p<0.01$ versus the H-RMR1X group; ${ }^{\dagger} p<0.05$ and ${ }^{\dagger \dagger} p<0.01$ versus the $H-D C 1 X$ group $(n=8)$.
\end{abstract}

higher protective factor HDL-C level and a lower risk factor LDL-C level. The results in Table 2 indicated that feeding a high cholesterol diet for 8 weeks would lead to an increase in the ratio of LDL-C to HDL-C as compared to that of the C group $(p>0.05)$. Therefore, the results obtained by a statistical analysis show that the ratio of LDL-C to HDL-C was less by $46.3 \%$ in the H-RMD1X group than in the H group $(p<0.01)$. RMR was able to perform a significant effect of $p<0.05$ on lowering the ratio of LDL-C to HDL-C by $45.1 \%$, but unfermented dioscorea was not $(p>0.05)$, as compared with the $\mathrm{H}$ group.

The Hypocholesterolemic and Hypotriglyceridemic Effect in Liver. The effect of RMD on lowering the TC and TG levels of liver was shown in Table 3. As expected, hamsters treated with a high cholesterol diet for 8 weeks showed a remarkable increase in liver TC and TG levels as compared to the $\mathrm{C}$ group $(p<0.01)$. A 1 -fold dose of RMD would decrease liver TC and TG levels with significant effects of $p<0.05$ and $p<0.01$, respectively. However, the significant hypocholesterolemic effect was unable to be expressed in RMR and the unfermented dioscorea treated group $(p>0.05)$. In addition, the RMR treatment but not the unfermented dioscorea treatment was able to perform a significant hypotriglyceridemic effect in liver $(p<0.05)$. Therefore, the statistical significances performed by 


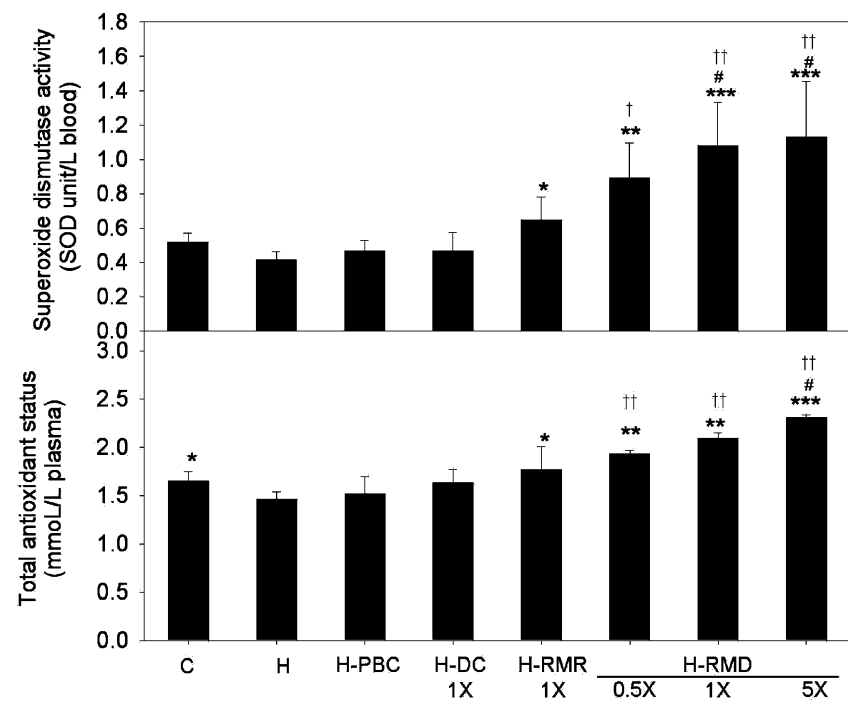

Figure 1. Effect of red mold dioscorea on SOD activity (A) and the total antioxidant status $(B)$ in the blood of hyperlipidemic hamsters. Two groups of the hamsters were fed a normal diet (the $\mathrm{C}$ group) or a high cholesterol diet (the $\mathrm{H}$ group) without the administration of test materials, respectively. The other hyperlipidemic hamsters were administrated with probucol (100 $\mathrm{mg} / \mathrm{kg} /$ day) (the H-PBC group), a l-fold dose of unfermented dioscorea (96 mg/kg/day) (the H-DC group), a l-fold dose of RMR (96 mg/kg/day including $0.83 \mathrm{mg}$ of monacolin $\mathrm{K}$ ) (the H-RMR1X group), a 0.5 -fold dose of RMD (48 mg/kg/day including $0.84 \mathrm{mg}$ of monacolin K) (the H-RMD0.5X group), a 1 -fold dose of RMD (96 mg/kg/day including $1.68 \mathrm{mg}$ of monacolin K) (the H-RMD1X group), and a 5-fold dose of RMD (480 $\mathrm{mg} / \mathrm{kg} /$ day including $8.4 \mathrm{mg}$ of monacolin K) (the H-RMD5X group). ${ }^{*} p<$ $0.05,{ }^{* *} p<0.01$, and ${ }^{* * *} p<0.001$ versus the H group; $\# p<0.05$, \#\# $p<$ 0.01 , and $\# \# p 0.001$ versus the RMR H-RMR1X group; ${ }^{\dagger} p<0.05$, ${ }^{\dagger \dagger} p$ $<0.01$, and ${ }^{\mathrm{tt}} p<0.001$ versus the H-DC1X group $(n=8)$.

RMD were higher than that performed by RMR or dioscorea based on the comparison with the $\mathrm{H}$ group.

Antiatherosclerotic Effect. SOD Activity and TAS. The oxidative stress in blood is associated with the development of atherosclerosis. Both SOD activity and TAS were usually used as the marks to evaluate the oxidative status in the atherosclerotic study (30). This study evaluated the effect of RMD on the SOD activity and TAS in the plasma of a hyperlipidemic hamster. As shown in Figure 1, feeding a high cholesterol diet for 8 weeks led to a decrease by $19.9 \%(p>0.05)$ and $11.6 \%(p<0.05)$ in SOD activity and TAS. However, the decreased antioxidative effect would be significantly reversed by RMD administration. Administration with a 0.5 -fold, a 1-fold, and a 5-fold dose of RMD would respectively significantly stimulate SOD activity by $115 \%(p<0.01), 160 \%(p<0.001)$, and $173 \%(p<0.001)$ as well as increase TAS by $32.7 \%(p<0.01), 43.6 \%(p<0.01)$, and $58.3 \%(p<0.001)$ with the dose-response, as compared with the H group. Importantly, a 1-fold dose of RMD would perform a higher SOD activity by $67.3 \%(p<0.05)$ and TAS by $18.3 \%$ $(p>0.05)$ than a 1-fold dose of RMR. Furthermore, unfermented dioscorea have been proven to express antioxidative ability well, which resulted in a weaker SOD activity (at least $p<0.05$ ) and TAS (at least $p<0.01$ ) than all doses of RMD. The above results clearly proved that RMD has a more antiatherosclerotic effect on increasing SOD activity of blood and TAS of plasma than RMR and unfermented dioscorea.

Catalase Activity. As shown in Figure 2, catalase activity between the serum and the liver had similar trends and a high relationship. The catalase activity of plasma and the liver of the $\mathrm{C}$ group were $12.70 \mathrm{U} / \mathrm{mg}$ protein and $10.6 \mathrm{U} / \mathrm{mg}$ protein,

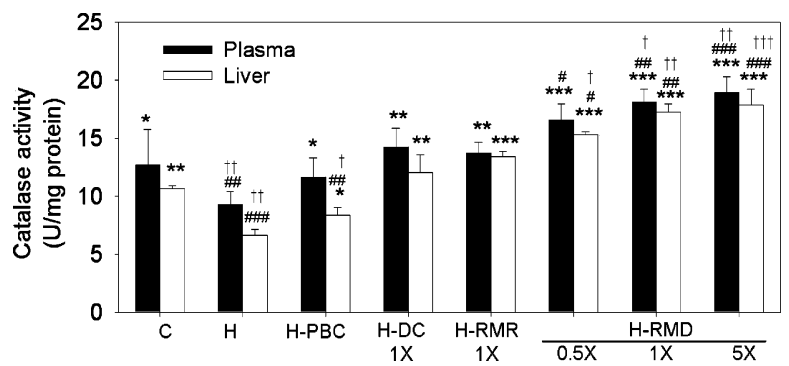

Figure 2. Effect of red mold dioscorea on the catalase activity of plasma and liver in hyperlipidemic hamsters. Two groups of the hamsters were fed a normal diet (the $\mathrm{C}$ group) or a high cholesterol diet (the $\mathrm{H}$ group) without the administration of test materials, respectively. The other hyperlipidemic hamsters were administrated with probucol (100 $\mathrm{mg} / \mathrm{kg} /$ day) (the H-PBC group), a l-fold dose of unfermented dioscorea (96 mg/kg/day) (the H-DC group), a l-fold dose of RMR (96 mg/kg/day including $0.83 \mathrm{mg}$ of monacolin $\mathrm{K}$ ) (the H-RMR1X group), a 0.5-fold dose of RMD (48 mg/kg/day including $0.84 \mathrm{mg}$ of monacolin $\mathrm{K}$ ) (the H-RMD0.5X group), a 1-fold dose of RMD (96 mg/kg/day including $1.68 \mathrm{mg}$ of monacolin K) (the H-RMD1X group), and a 5-fold dose of RMD (480 $\mathrm{mg} / \mathrm{kg} /$ day including $8.4 \mathrm{mg}$ of monacolin $\mathrm{K}$ ) (the H-RMD5X group). ${ }^{*} p<$ $0.05,{ }^{* *} p<0.01$, and ${ }^{* * *} p<0.001$ versus the $\mathrm{H}$ group; ${ }^{*} p<0.05$, ${ }^{\# \#} p<$ 0.01 , and ${ }^{\# \#} p<0.001$ versus the RMR H-RMR1X group; ${ }^{\dagger} p<0.05$, ${ }^{\dagger \dagger} p$ $<0.01$, and ${ }^{t+t} p<0.001$ versus the H-DC1X group $(n=8)$.

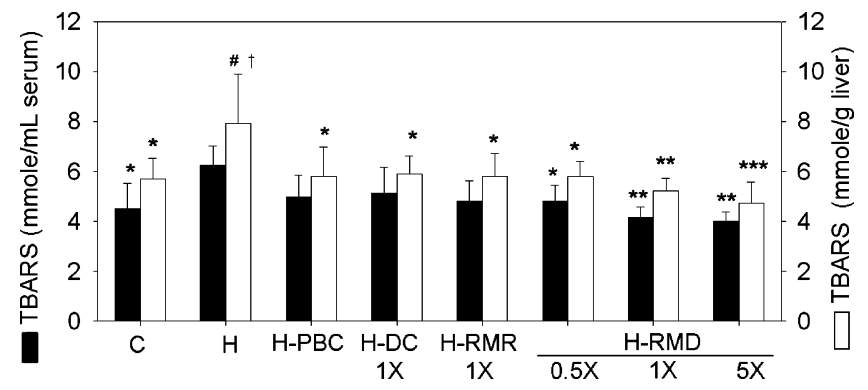

Figure 3. Effect of red mold dioscorea on lipid peroxidation in the blood and the liver of hyperlipidemic hamsters. Two groups of the hamsters were fed a normal diet (the $\mathrm{C}$ group) or a high cholesterol diet (the $\mathrm{H}$ group) without the administration of test materials, respectively. The other hyperlipidemic hamsters were administrated with probucol (100 $\mathrm{mg} / \mathrm{kg} /$ day) (the H-PBC group), a l-fold dose of unfermented dioscorea (96 mg/kg/day) (the H-DC group), a l-fold dose of RMR (96 mg/kg/day including $0.83 \mathrm{mg}$ of monacolin $\mathrm{K}$ ) (the H-RMR1X group), a 0.5-fold dose of RMD (48 mg/kg/day including $0.84 \mathrm{mg}$ of monacolin $\mathrm{K}$ ) (the H-RMD0.5X group), a 1-fold dose of RMD (96 mg/kg/day including $1.68 \mathrm{mg}$ of monacolin K) (the H-RMD1X group), and a 5-fold dose of RMD (480 $\mathrm{mg} / \mathrm{kg} /$ day including $8.4 \mathrm{mg}$ of monacolin K) (the H-RMD5X group). " $p<$ $0.05,{ }^{* *} p<0.01$, and ${ }^{* * *} p<0.001$ versus the $\mathrm{H}$ group; $" \#<0.05$, $\# p<$ 0.01 , and ${ }^{\# \#} p<0.001$ versus the RMR H-RMR1X group; ${ }^{\dagger} p<0.05,{ }^{\dagger+} p$ $<0.01$, and $\mathrm{tt}^{\dagger} p<0.001$ versus the H-DC1X group $(n=8)$.

respectively, which would be decreased to $9.30 \mathrm{U} / \mathrm{mg}$ protein $(p<0.05)$ and $6.61 \mathrm{U} / \mathrm{mg}$ protein $(p<0.01)$ by feeding a high cholesterol diet for 8 weeks. H-RMR1X and H-DC groups both performed a significantly higher catalase activity of plasma and liver than the H-group (at least $p<0.01$ ) but less than the H-RMD1X group (at least $p<0.05$ ). The catalase activity of plasma and liver in a hyperlipidemic hamster would be increased by RMD administration with the dose-response and significant difference of $p<0.001$.

Lipid Peroxidation. TBARS assay was the general procedure for the evaluation of lipid peroxidation. In the results of Figure 3, feeding a high cholesterol diet for 8 weeks caused the $\mathrm{H}$ group serious lipid peroxidation in serum and liver $(p<0.05$ vs the $\mathrm{H}$ 


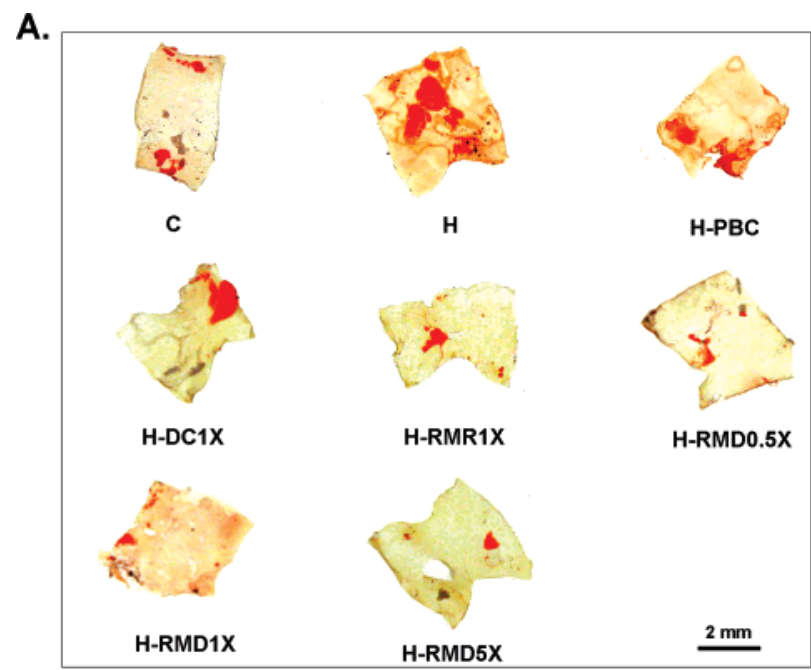

B.

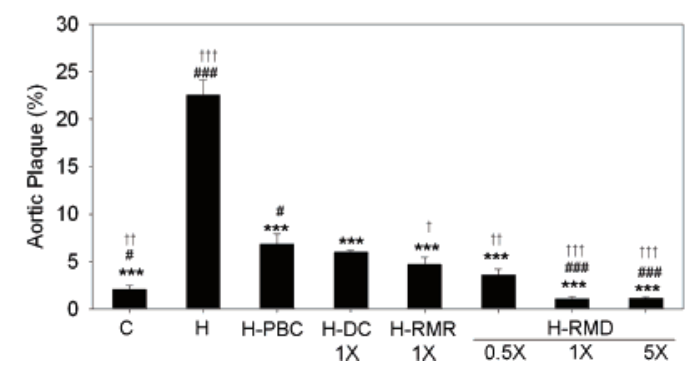

Figure 4. Effect of red mold dioscorea on the atherosclerotic plaque in the thoracic aorta of hyperlipidemic hamsters: $(A)$ the atherosclerotic plaque presented as the red dye in the graph and $(B)$ the proportion of the area of the atherosclerotic plaque in the aorta. The whole surface area of the thoracic aorta was stained by Sudan IV and photographed using a digital camera. The aortic surface area and its stained plaque area (red dye) were selected and quantitated by the Posterize program of Photoshop 7.0 software. The selected pixel of the plaque area and the whole aorta was used to calculate the percent area of the aortic plaque. Two groups of the hamsters were fed a normal diet ( $C$ group) or a high cholesterol diet ( $\mathrm{H}$ group) without the administration of test materials, respectively. The other hyperlipidemic hamsters were administrated with probucol (100 mg/kg/day) (the H-PBC group), a l-fold dose of unfermented dioscorea (96 mg/kg/day) (the H-DC group), a l-fold dose of RMR (96 $\mathrm{mg} / \mathrm{kg} /$ day including $0.83 \mathrm{mg}$ of monacolin $\mathrm{K}$ ) (the H-RMR1X group), a 0.5 -fold dose of RMD (48 mg/kg/day including $0.84 \mathrm{mg}$ of monacolin $\mathrm{K}$ ) (the H-RMD0.5X group), a 1-fold dose of RMD (96 mg/kg/day including $1.68 \mathrm{mg}$ of monacolin K) (the H-RMD1X group), and a 5 -fold dose of RMD (480 mg/kg/day including $8.4 \mathrm{mg}$ of monacolin $\mathrm{K}$ ) (the H-RMD5X group). ${ }^{*} p<0.05,{ }^{* *} p<0.01$, and ${ }^{* * *} p<0.001$ versus the $H$ group; ${ }^{*} p<$ $0.05,{ }^{\#} p<0.01$, and ${ }^{\# \#} p<0.001$ versus the RMR H-RMR1X group; ${ }^{\dagger} p$ $<0.05,{ }^{\dagger \dagger} p<0.01$, and ${ }^{t+t} p<0.001$ versus the H-DC1X group $(n=8)$.

group). The RMD treatment would decrease the TBARS levels of serum and liver in a hyperlipidemic hamster, and the statistical result would be more significant with the dose-response (at least $p<0.01$ vs the $\mathrm{H}$ group). A half-fold dose of the RMD treatment resulted in a statistical significance of $p<0.05$, however, which was also performed in the serum but not the liver of the H-RMR1X and H-DC groups. The degree of TBARS would be relieved by the RMD treatment with the doseresponse and significant effect.

Atherosclerotic Plaque of Aorta. Atherosclerotic plaque in aorta caused by oxidative stress and lipid accumulation is the atheromatous lesions in atherosclerosis. As illustrated in Figure 4, the lipid plaque would be largely accumulated at $22.5 \%$ in the aorta of hyperlipidemic hamster. RMR and dioscorea treatment resulted in a significant decrease by $79.1 \%$ and $73.3 \%$ in the percent area of the atheromatous lesions as compared with the $\mathrm{H}$ group. The RMD treatment trended to decrease the percent area of the atheromatous lesions with the dose-response. The percent area of aortic plaque would be significantly decreased to $1.05 \%$ and $1.10 \%$ by administration with 1 -fold and 5-fold doses of RMD, respectively.

\section{DISCUSSION}

Atherosclerosis constitutes the most common medical problem. This can be manifested clinically as a stroke, coronary artery disease, or peripheral vascular disease (31). The causal relationship between blood cholesterol level and atherosclerosis was widely accepted, as there was already considerable interest in the possibility of using drugs to decrease cholesterol levels. The Monascus species is proven to produce the 3-hydroxy-3methylglutaryl-coenzyme A (HMG-CoA) reductase inhibitor, monacolin $\mathrm{K}$, so that its fermented rice has been applied to the commercial functional food with the hypolipidemic effect $(5$, 6). Therefore, monacolin $\mathrm{K}$ is regarded as the most important active compound for the hypolipidemic effect in the Monascus fermented product. Many studies tested various fermentation conditions of the Monascus species in order to raise the production of monacolin $\mathrm{K}(32,33)$. Our previous study used dioscorea to ferment the Monascus species and gained the RMD including high monacolin $\mathrm{K}$ production (7). Dioscorea is also proven to perform potential the hypolipidemic effect and antioxidative ability by many studies $(10,12)$. Therefore, the goal of the study is to develop a novel Monascus functional food with a more potent hypolipidemic and antiatherosclerotic effect.

Both RMR and dioscorea are proven to perform a potent hypolipidemic effect in previous studies $(3,5,12)$, but RMD may be found as the novel product with a more potent hypolipidemic effect in this study. A 1-fold dose of RMR had performed a significant hypocholesterolemic effect of $p<0.01$ and a hypotriglyceridemic effect of $p<0.05$ in serum results, but the same dose of RMD would result in a more significant hypocholesterolemic effect of $p<0.001$ and a hypotriglyceridemic effect of $p<0.01$, as compared with the $\mathrm{H}$ group. Furthermore, the effect on lowering the ratio of LDL-C to HDL-C was preformed more significantly in a 1-fold dose of RMD ( $p<0.01$ versus the H group) than in the same dose of RMR ( $p<0.05$ versus $H$ the group). Therefore, the result can suggest that RMD had a greater hyperlipidemic effect than RMR. RMD includes a higher monacolin K concentration by 2-fold than RMR, which should be one of the reasons why RMD has a more hypocholesterolemic effect than RMR. In regard to the hypolipidemic effect of unfermented dioscorea, dioscorea had no statistical significant effect on lowering the serum TG, LDL-C, and LDL-C/HDL-C ratio besides TC, but RMD and RMR were able to respectively lead to a significant effect (at least $p<0.01$ and $p<0.05$ versus $\mathrm{H}$ the group) on these hypolipidemic markers. Furthermore, the RMR had a higher significant effect (at least $p<0.05$ versus the $\mathrm{H}$ group). According to the statistical effects versus the $\mathrm{H}$ group, the hypolipidemic effect order is successive: RMD, RMR, unfermented dioscorea.

Although RMD has been proven to exhibit a greater hypolipidemic effect in this study, the dose-response was unobvious. According to the results, the more potent effect can be observed as the treated dose was increased from a half-fold to 1-fold, but the effect was unable to be stronger as the dose was increased to 5-fold. The unobvious effect is possible in that 
RMD had performed the most activity in the hypocholesterolemic and hypotriglyceridemic effect. However, the assimilation and bioavailability of the high dose of RMD may be an obvious factor for the dose-response. The in vivo bioavailability of the complex such as food, herb, and fermented product was a concern of many studies $(34,35)$. RMD consists of many and multiple materials including Monascus metabolites and dioscorea starch and ingredients so high that an amount of RMD was possible to be completely assimilated and used by the digestive system. Furthermore, the regulation of cholesterol biosynthesis may affect the effect of RMD. This study is to investigate the hypolipidemic effect of RMD, but the above problem may affect the effect of the dose-response and should be resolved in the future study of RMD or the Monascus product.

Dioscorea has been proven to exhibit a strong antioxidative ability as well as repress hyperlipidemia-induced oxidative stress (12). Oxidative stress is an important risk factor in the pathogenesis of atherosclerosis (13). The involvement of ROS in the oxidative modification of LDL, an important risk factor of atherogenesis, has drawn attention to the antioxidative defense of ROS scavengers in the organism. The antioxidative enzymes, so-called primary scavengers, in red blood cells and serum include SOD, seleno-dependent glutathione peroxidase, glutathione reductase, and catalase (36). The highly aggressive and toxic hydroxyl radicals have been proposed to play important pathogenic roles involved in attacking lipids, proteins, or DNA to acquire the missing electron. However, catalase conjugated to SOD would repress the conversion of hydrogen peroxide to hydroxyl radicals. Superoxide anion radicals are dismuted by SOD to hydrogen peroxide and oxygen. Under physiological conditions, the conjugation of catalase to SOD ensures that as soon as a superoxide dismutation reaction occurs, the resultant $\mathrm{H}_{2} \mathrm{O}_{2}$ is removed by the immediate proximity of the catalase molecule (36). A high LDL-C level certainly leads to both a decreased activity of antioxidative mechanisms and an excess of substrate, which can undergo abnormal oxidative modifications (13). According to the above suggestion, this study found that RMD has a great effect to lower the LDL-C level, which would be an advantage to prevent hyperlipidemia-induced oxidative stress in blood and liver. The increase of TAS, SOD, and catalase activity and the repression of lipid oxidation in the blood or the liver of hyperlipidemic hamsters were exhibited by the RMD treatment, suggesting that the antioxidative ability performed by RMD was able to resist hypolipidemia-induced oxidative stress. The formation of atherosclerotic plaque is proven to be increased by increasing the level of ox-LDL and oxidative stress of the blood circulation $(13,37)$. The result of this study also found that the there was a high relationship between serum LDL-C levels and atherosclerotic plaque formation. The potent effect of RMD on repressing the formation of atherosclerotic plaque can result from the LDL-C-lowering effect and antioxidative effect.

Both RMR and dioscorea also exhibit significant protection against oxidative stress, but the effect would be less than the RMD treatment. Therefore, a more potent antiatherosclerotic effect performed by RMD should be attributed to the contribution of dioscorea and Monascus metabolite. Hypolipidemic Monascus metabolites include sterols ( $\beta$-sitosterol, campesterol, stigmasterol, and sapogenin), isoflavones and isoflavone glycosides, and monounsaturated fatty acids in addition to monacolin K (6). Furthermore, the antioxidative ability of the Monascus fermented product is attributed to dimerumic acid, tannin, and phenol $(38,39)$. In addition, monascin, a yellow pigment of RMD with anti-inflammatory ability, may contribute to the repression of an oxidized cholesterol-induced inflammatory response and atherosclerotic plaque. Dioscorea, a substrate of RMD, has been widely used as a functional food and Chinese herb for the treatment of gastrointestinal disease (40). The functional compounds of dioscorea including resistance starch, polysaccharides, flavones, vitamin $\mathrm{C}$, polyphenol, and sporamin are related to the performance of the antioxidative ability $(9-12,41)$. In addition, dioscin and diosgenin of dioscorea are proven to repress an inflammatory response and lower TC and TG levels (12). These healthy functions of dioscorea and Monascus metabolite should augment the effect of RMD on the hypolipidemic and antiatherosclerotic effect.

In conclusion, the study compared the hypolipidemic and antiatherosclerotic effect with RMD, RMR, and unfermented dioscorea. In the results, only needing a 0.5 -fold dose of RMD was able to significantly lower the total cholesterol, triglyceride, and low-density lipoprotein cholesterol levels as well as maintain a high-density lipoprotein cholesterol (HDL-C) level. RMD provided more hypolipidemic effect than RMR and unfermented dioscorea, resulting from higher monacolin K levels of RMD. Regarding the prevention of arteriosclerosis, unfermented dioscorea was able to exhibit potent antioxidative ability and protection against hyperlipidemia-induced oxidative stress. The effect further strengthened the treatment of RMD for stimulating blood and liver antioxidase activity and repressing lipid peroxidation and atherosclerotic plaque. RMD combined the potent hypolipidemic effect of Monascus metabolites and the potent antioxidative ability of dioscorea and was able to perform a more significant hypolipidemic and antiatherosclerotic effect than RMR and dioscorea.

\section{LITERATURE CITED}

(1) Steinberg, H. O.; Bayazeed, B.; Hook, G.; Johnson, A.; Cronin, J.; Baron, A. D. Endothelial dysfunction is associated with cholesterol levels in the high normal range in humans. Circulation 1997, 96, 3287-3293.

(2) Yla-Herttuala, S.; Palinski, W.; Rosenfeld, M. E.; Parthasarathy, S.; Carew, T. E.; Butler, S.; Witztum, J. L.; Steinberg, D. Evidence for the presence of oxidatively modified low density lipoprotein in atherosclerotic lesions of rabbit and man. J. Clin. Invest. 1989, 84, 1086-1095.

(3) Lee, C. L.; Tsai, T. Y.; Wang, J. J.; Pan, T. M. In vivo hypolipidemic effects and safety of low dosage Monascus powder in a hamster model of hyperlipidemia. Appl. Microbiol. Biotechnol. 2006, 70, 533-540.

(4) Li, C. L.; Zhu, Y.; Wang, Y. Y.; Zhu, J. S.; Chang, J.; Kritchevsky, D. Monascus purpureus-fermented rice (red yeast rice): a natural food product that lowers blood cholesterol in animal models of hypercholesterolemia. Nutr. Res. 1998, 18, 7181.

(5) Wei, W.; Li, C.; Wang, Y.; Su, H.; Zhu, J.; Kritchevsky, D. Hypolipidemic and anti-atherogenic effects of long-term Cholestin (Monascus purpureus-fermented rice, red yeast rice) in cholesterol fed rabbits. J. Nutr. Biochem. 2003, 14, 314-318.

(6) Heber, D.; Yip, I.; Ashley, J. M.; Elashoff, D. A.; Elashoff, R. M.; Go, V. L. Cholesterol-lowering effects of a proprietary Chinese red-yeast-rice dietary supplement. Am. J. Clin. Nutr. 1999, 69, 231-236.

(7) Lee, C. L.; Wang, J. J.; Kuo, S. L.; Pan, T. M. Monascus fermentation of dioscorea for increasing the production of cholesterol-lowering agent-monacolin $\mathrm{K}$ and antiinflammation agent-monascin. Appl. Microbiol. Biotechnol. 2006, 72, 12541262.

(8) Zernecke, A.; Weber, C. Inflammatory mediators in atherosclerotic vascular disease. Basic Res. Cardiol. 2005, 100, 93-101. 
(9) Wanasundera, J. P.; Ravindran, G. Nutritional assessment of yam (Dioscorea alata) tubers. Plant Foods Hum. Nutr. (Dordrecht, Neth.) 1994, 46, 33-39.

(10) Chang, S. J.; Lee, Y. C.; Liu, S. Y.; Chang, T. W. Chinese yam (Dioscorea alata cv. Tainung No. 2) feeding exhibited antioxidative effects in hyperhomocysteinemia rats. J. Agric. Food Chem. 2004, 52, 1720-1725.

(11) Nagai, T.; Nagashima, T. Functional properties of dioscorin, a soluble viscous protein from Japanese yam (Dioscorea opposita thunb.) tuber mucilage Tororo. Z. Naturforsch., C: J. Biosci. 2006, 61, 792-798.

(12) Chang, W. C.; Yu, Y. M.; Wu, C. H.; Tseng, Y. H.; Wu, K. Y. Reduction of oxidative stress and atherosclerosis in hyperlipidemic rabbits by Dioscorea rhizome. Can. J. Physiol. Pharmacol. 2005, 83, 423-430.

(13) Jessup, W.; Dean, R. T.; de Whalley, C. V.; Rankin, S. M.; Leake, D. S. The role of oxidative modification and antioxidants in LDL metabolism and atherosclerosis. Adv. Exp. Med. Biol. 1990, 264, 139-142.

(14) Su, Y. C.; Wang, J. J.; Lin, T. T.; Pan, T. M. Production of the secondary metabolites gamma-aminobutyric acid and monacolin K by Monascus. J. Ind. Microbiol. Biotechnol. 2003, 30, 4146.

(15) Boyd, E. The growth of the surface area of human body; University of Minnesota Press: 1935.

(16) Wang, W. H.; Zhang, H.; Yu, Y. L.; Ge, Z.; Xue, C.; Zhang, P. Intervention of xuezhikang on patients of acute coronary syndrome with different levels of blood lipids. Zhongguo Zhong Xi Yi Jie He Za Zhi 2004, 24, 1073-1076.

(17) Aubin, M. C.; Carrier, M.; Shi, Y. F.; Tardif, J. C.; Perrault, L. P. Role of probucol on endothelial dysfunction of epicardial coronary arteries associated with left ventricular hypertrophy. J. Cardiovasc. Pharmacol. 2006, 47, 702-710.

(18) Usman; Hosono, A. Hypocholesterolemic effect of Lactobacillus gasseri SBT0270 in rats fed a cholesterol-enriched diet. J. Dairy Res. 2001, 68, 617-624.

(19) Friedewald, W. T.; Levy, R. I.; Fredrickson, D. S. Estimation of the concentration of low-density lipoprotein cholesterol in plasma, without use of the preparative ultracentrifuge. Clin. Chem. 1972, 18, 499-502.

(20) Schimke, I.; Romaniuk, P.; Schimke, E.; Papies, B. Concentration of thiobarbituric acid-reactive substances (TBARS) in the plasma of patients with atherosclerosis with different localizations and different degrees of severity. Z. Med. Lab. Diagn. 1990, 31, 176180.

(21) Mabile, L.; Fitoussi, G.; Periquet, B.; Schmitt, A.; Salvayre, R.; Negre-Salvayre, A. alpha-Tocopherol and trolox block the early intracellular events (TBARS and calcium rises) elicited by oxidized low-density lipoproteins in cultured endothelial cells. Free Radical Biol. Med. 1995, 19, 177-187.

(22) Ohkawa, H.; Ohishi, N.; Yagi, K. Assay for lipid peroxides in animal tissues by thiobarbituric acid reaction. Anal. Biochem. 1979, 95, 351-358.

(23) Miller, N. J.; Rice-Evans, C.; Davies, M. J.; Gopinathan, V.; Milner, A. A novel method for measuring antioxidant capacity and its application to monitoring the antioxidant status in premature neonates. Clin. Sci. (London) 1993, 84, 407-412.

(24) Beers, R. F. J.; Sizer, I. W. A spectrophotometric method for measuring the breakdown of hydrogen peroxide by catalase. $J$. Biol. Chem. 1952, 195, 133-140.

(25) Lee, C. L.; Wang, J. J.; Pan, T. M. Synchronous analysis method for detection of citrinin and the lactone and acid forms of monacolin $\mathrm{K}$ in red mold rice. J. AOAC Int. 2006, 89, 669677.

(26) Lin, C. F.; Iizuka, H. Production of Extracellular Pigment by a Mutant of Monascus kaoliang sp. nov. Appl. Environ. Microbiol. 1982, 43, 671-676.

(27) Aniya, Y.; Yokomakura, T.; Yonamine, M.; Shimada, K.; Nagamine, T.; Shimabukuro, M.; Gibo, H. Screening of antioxidant action of various molds and protection of Monascus anka against experimentally induced liver injuries of rats. Gen. Pharmacol. 1999, 32, 225-231.

(28) Oyaizu, M. Antioxidative activity of browning products of glucosamine fractionated by organic solvent and thin-layer chromatography. Nippon Shokuhin Kogyo Gakkaishi 1986, 35, $771-775$.

(29) Lingnert, H.; Vallentin, K.; Eriksson, C. E. Measurement of antioxidative in model system. J. Food Process. Preserv. 1979. 3, 87-103.

(30) Zawadzka-Bartczak, E. Activities of red blood cell anti-oxidative enzymes (SOD, GPx) and total anti-oxidative capacity of serum (TAS) in men with coronary atherosclerosis and in healthy pilots. Med. Sci. Monit. 2005, 11, CR440-444.

(31) Kratz, M. Dietary cholesterol, atherosclerosis and coronary heart disease. Handb. Exp. Pharmacol. 2005, 170, 195-213.

(32) Chen, F.; Hu, X. Study on red fermented rice with high concentration of monacolin $\mathrm{K}$ and low concentration of citrinin. Int. J. Food Microbiol. 2005, 103, 331-337.

(33) Wang, J. J.; Lee, C. L.; Pan, T. M. Improvement of monacolin $\mathrm{K}$, gamma-aminobutyric acid and citrinin production ratio as a function of environmental conditions of Monascus purpureus NTU 601. J. Ind. Microbiol. Biotechnol. 2003, 30, 669-676.

(34) Zhu, M.; Wong, P. Y.; Li, R. C. Effects of taraxacum mongolicum on the bioavailability and disposition of ciprofloxacin in rats. J. Pharm. Sci. 1999, 88, 632-634.

(35) Yan, R.; Lin, G.; Ko, N. L.; Tam, Y. K. Low oral bioavailability and pharmacokinetics of senkyunolide a, a major bioactive component in Rhizoma Chuanxiong, in the rat. Ther. Drug Monit. 2007, 29, 49-56.

(36) Liczmanski, A. E. Oxygen toxicity. II. Defense mechanisms. Postepy Biochem. 1988, 34, 293-310.

(37) Baskin, Y.; Baskin, H.; Guner, G.; Tuzun, E.; Oto, O. The inversely proportional relation between nitric oxide and lipid peroxidation in atherosclerotic plaque formation in human. Int. J. Cardiol. 2003, 91, 53-57.

(38) Juzlova, P.; Martinkova, L.; Kren, V. Secondary metabolites of the fungus Monascus: a review. J. Ind. Microbiol. 1996, 16, $163-170$.

(39) Rhyu, M. R.; Kim, D. K.; Kim, H. Y.; Kim, B. K. Nitric oxidemediated endothelium-dependent relaxation of rat thoracic aorta induced by aqueous extract of red rice fermented with Monascus ruber. J. Ethnopharmacol. 2000, 70, 29-34.

(40) Jeon, J. R.; Lee, J. S.; Lee, C. H.; Kim, J. Y.; Kim, S. D.; Nam, D. H. Effect of ethanol extract of dried Chinese yam (Dioscorea batatas) flour containing dioscin on gastrointestinal function in rat model. Arch. Pharm. Res. 2006, 29, 348-353.

(41) Misaki, A.; Ito, T.; Harada, T. Constitutional studies on the mucilage of yamanoimo, Dioscorea batatas Decne, forma Tsukune. Isolation and structure of a mannan. Agric. Biol. Chem. 1972, 36, 761-771.

Received for review May 3, 2007. Revised manuscript received June 19, 2007. Accepted June 21, 2007.

JF071293J 